\title{
Mentalidad Resiliente y Salud Mental Positiva en docentes de Nivel Superior
}

\begin{abstract}
Linda Miriam Silerio Hernández ${ }^{a}$, Arturo Barraza Macías ${ }^{b}$, Dora Luz González-Bañales ${ }^{c}$
${ }^{a}$ Departamento de Ciencias Básicas, Instituto Tecnológico de Durango, Tecnológico Nacional de México, Durango, México, sihelinda@hotmail.com; ${ }^{\mathrm{b}}$ Universidad Pedagógica de Durango, México, praxisredie2@gmail.com; ${ }^{c}$ Departamento de Sistemas y Computación, Instituto Tecnológico de Durango, Tecnológico Nacional de México, Durango, México, doraglez@itdurango.edu.mx
\end{abstract}

\section{Resumen}

El objetivo general es analizar la relación existente entre Mentalidad Resiliente (caracteristicas de las personas que logran sobreponerse con éxito ante adversidades y logran ver en estas situaciones oportunidades de crecimiento) y Salud Mental Positiva (conjunto de características de personalidad y habilidades bio-psico-sociales que una persona tiene para alcanzar metas vitales y de autorrealización), en docentes de nivel superior. Se utilizó el método hipotético deductivo con encuestas, aplicado a 238 docentes de siete instituciones públicas más importantes en la ciudad de Durango, México. La evidencia encontrada sugiere que los docentes desarrollan frecuentemente una Mentalidad Resiliente, a través de mostrar diálogos de vida, empatía, comunicación, aceptarse a sí mismos y a los demás, establecer contactos y mostrar compasión, manejar errores, manejar el éxito, desarrollar autodisciplina y autocontrol y conocer las características de una vida resiliente para mejorar su estilo de vida, también desarrollan frecuentemente una Salud Mental Positiva, a través del bienestar cognitivo emocional, dominar el entorno, tener habilidades sociales, tener bienestar fisico y autorreflexionar. Se realizaron análisis correlacionales.

Palabras clave: Mentalidad Resiliente, Salud Mental Positiva, Profesores de Educación Superior. 


\section{Introducción}

Dada la importancia de optimizar el proceso enseñanza-aprendizaje en los docentes de Nivel Superior de la Ciudad de Durango, Dgo., México, es de vital importancia que éstos puedan desarrollar una Salud Mental Positiva y una Mentalidad Resiliente que les permita enfrentar las situaciones adversas en el área personal, familiar, social y ambiental y gozar de una vida resiliente, mentalmente sana.

El desarrollo de esta investigación está orientado desde la perspectiva positiva de Salud Mental, definida como "un conjunto de características de personalidad y habilidades bio-psicosociales que una persona tiene para alcanzar metas vitales y de autorrealización, estados de bienestar y adaptación a su contexto" (Barrera \& Flores 2013) y la Mentalidad Resiliente como un componente del ser humano que se utiliza para desarrollar la Resiliencia a lo largo de la vida, para superar el dolor, el estrés y/o la adversidad (Brooks \& Goldstein, 2010).

El término Resiliencia se presenta como un constructo personal a partir de las investigaciones previas, que hace referencia a las características de las personas que logran sobreponerse con éxito ante la crisis, adversidades o situaciones traumáticas y logran ver en estas situaciones oportunidades de crecimiento, fortaleza y desarrollo personal.

Los objetivos de la presente investigación están orientados a determinar la relación existente entre la Mentalidad Resiliente y la Salud Mental Positiva en los docentes de nivel superior y los niveles de ambos constructos en los docentes de nivel superior de la ciudad de Durango, Dgo. México.

\section{Marco Teórico: Resiliencia y Salud Mental}

Dentro de los principales aportes teóricos relacionados con Resiliencia se pueden mencionar los siguientes: a) Teoría de las siete resiliencias (Wolin \&Wolin, 1993), b) Modelo de verbalizaciones de Edith Henderson Grotberg (1996), c) Modelo de la Casita de Stefan Vanistendael (2002), d) Modelo de Resiliencia de Boris Cyrulnik (2002), Modelo de la Rueda de la Resiliencia de Henderson y Milstein (2003), e) Referente teórico del Poder de la Resiliencia de Robert Brooks y Sam Goldstein (2010).

En lo que respecta a Salud Mental se destacan los siguientes modelos: a) Modelo de Salud Mental desde la perspectiva negativa (OMS, 1990), b) Modelo de Salud Mental Positiva (OMS, 2004), c) Modelo conceptual de Salud Mental Positiva de Marie Jahoda (1958), d) Aporte teórico conceptual de Ma. Teresa Luch Canut (1999), e) Aporte teórico conceptual de Habilidades Fundamentales de Salud Mental Positiva de Tengland (2001), f) Modelo de Salud 
Mental desde la perspectiva positiva de Martín Seligman (2002), g) Aporte teórico de siete dimensiones de Salud Mental Positiva, para el diseño de un instrumento de Mónica Lolbé Barrera Guzmán (2011), h) Aporte teórico de la construcción de una escala de Salud Mental Positiva para adultos de población mexicana (Barrera \& Flores, 2013).

A continuación se presenta la propuesta de investigación del Modelo Comprehensivo Integral de Resiliencia y Salud Mental Positiva, a partir de las aportaciones teóricas anteriores. donde se plantea la relación entre Resiliencia, Mentalidad Resiliente y Salud Mental Positiva en donde se hipotetiza que hay una relación unidireccional donde la Mentalidad Resiliente influye sobre la Salud Mental Positiva, la cual será contrastada en la presente investigación (Fig. 1).

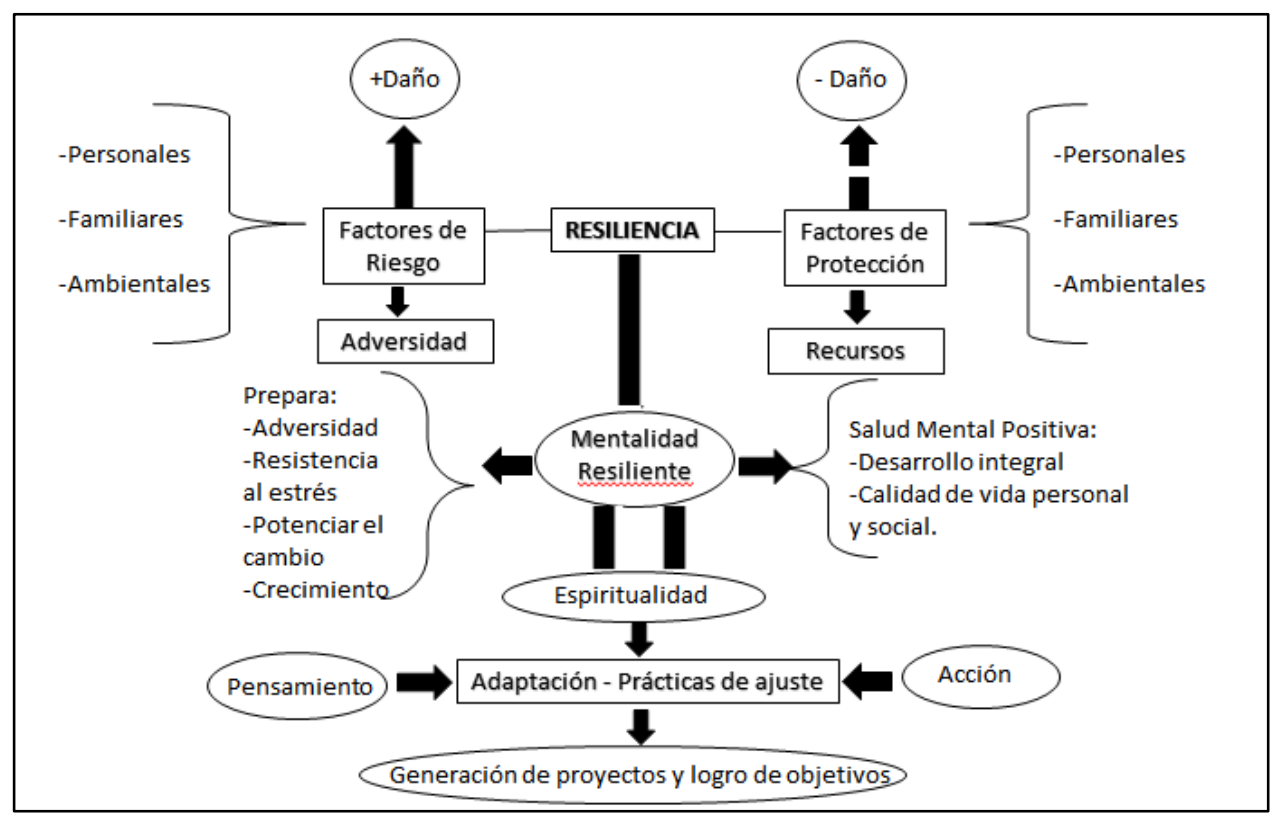

Fig. 1 Modelo Comprehensivo Integral de Mentalidad Resiliente y Salud Mental Positiva Fuente: Elaboración propia 


\section{Diseño Metodológico}

El enfoque de la presente investigación es cuantitativo, el método es hipotético - deductivo, el diseño no experimental, transversal, descriptivo y correlacional; la población está integrada por 1975 docentes de siete instituciones públicas de nivel superior de la ciudad de Durango, Dgo., la muestra seleccionada es de 238 docentes, la técnica utilizada es la encuesta y el instrumento de medición el cuestionario. La validez que presenta el instrumento de medición de la variable Mentalidad Resiliente tiene un valor medio numérico de 2.67 y se considera como fuerte. La confiabilidad que presenta el cuestionario de Mentalidad Resiliente es de .916 considerada como elevada, el cuestionario de Salud Mental Positiva presenta una confiabilidad de .883, en ambos casos considerada como elevada (Hernández, Fernández \& Baptista, 2010).

Las variables investigativas: La variable teórica 1: Mentalidad Resiliente definida como "componente del ser humano que permite el desarrollo de la Resiliencia a lo largo de la vida, para superar el dolor, el estrés y/o la adversidad y lograr el éxito". Está integrada por 9 dimensiones: diálogos de vida, empatía, comunicación, aceptación a sí mismo y a los demás, contactos y compasión, manejo de errores, manejo de éxito, autodisciplina y autocontrol, y conocimiento sobre una vida resiliente. La variable teórica 2: Salud Mental Positiva definida como "un conjunto de características de personalidad y habilidades bio-psico-sociales que una persona tiene para alcanzar metas vitales y de autorrealización, estados de bienestar y adaptación a su contexto" (Barrera \& Flores, 2013). Está integrada por 6 dimensiones: bienestar cognitivo emocional, dominio del entorno, habilidades sociales, empatía, bienestar físico y autorreflexión

Las preguntas en el cuestionario de este estudio son de respuesta a escala de Likert y están dirigidas a medir el nivel de las variables: Mentalidad Resiliente y Salud Mental Positiva, así como su relación. La escala tipo Likert está integrada por un conjunto de reactivos que se presentaron como afirmaciones, ante las cuales, se les pidió a los sujetos de investigación elegir una de las cuatro opciones de la escala que se les administró: siempre, frecuentemente, pocas veces y nunca. La versión final del cuestionario está integrada por la descripción de tres variables sociodemográficas, tres variables situacionales, 29 ítems de Mentalidad Resiliente y 30 de Salud Mental Positiva, éstos últimos fueron seleccionados con base al mayor peso factorial que presentan en el referente teórico de Barrera y Flores (2013). 


\section{Análisis de resultados}

En términos generales, los docentes de nivel superior participantes en el estudio, presentan respuestas medias en la variable Mentalidad Resiliente que tienden a 3.22 con una desviación estándar de 0.70, lo que sugiere que frecuentemente desarrollan este componente del ser humano. La dimensión que presenta el promedio más alto 3.46 es Aceptación a sí mismo y a los demás, lo que sugiere que frecuentemente realizan esta aprobación; la puntuación más baja 2.74 corresponde al ítem: "Conocimiento de una vida resiliente", aunque tiende a 3 , refleja la necesidad de conocer más acerca de las características de una vida resiliente para convertirse en su diario vivir en docentes promotores de Resiliencia, a través del diseño e implementación de programas de Resiliencia.

En términos generales la media de la variable Salud Mental Positiva, que presentan los docentes de nivel superior de la ciudad de Durango, Dgo. (México), es de 3.25 con una desviación estándar de 0.78, lo que sugiere que frecuentemente éstos, presentan una Salud Mental Positiva. La media más alta 3.39 corresponde a la dimensión Bienestar cognitivo emocional y la más baja 3.00 corresponde a la dimensión Bienestar físico, lo que refleja que los docentes poseen frecuentemente actitudes, cogniciones, emociones y características de personalidad que generan estados de ánimo agradables, de armonía integral, optimismo y una percepción de satisfacción con la vida y con la propia persona, sin embargo, pueden mejorar su estado de salud para sentirse físicamente bien y lograr un equilibrio físico, emocional y psicológico.

Se efectuó un análisis de correlación bivariada, dada la naturaleza no paramétrica de los datos se aplicó el Análisis Correlacional Rho de Spearman. Los resultados de esta correlación más alta entre las dimensiones de las dos variables presenta un valor de 495 (moderada) y se presenta entre establecer contactos y mostrar compasión de la variable Mentalidad Resiliente y Habilidades Sociales de la variable Salud Mental Positiva; la correlación más baja con un valor de .169 (débil) se presenta entre la dimension Comunicación de la variable Mentalidad Resiliente y Bienestar Físico de la variable Salud Mental Positiva (Tabla 1). 
Tabla 1 Correlación por dimensiones de las variables Mentalidad Resiliente y Salud Mental Positiva

Dimensiones Salud Mental Positiva

\begin{tabular}{|c|c|c|c|c|c|c|c|}
\hline \multirow{19}{*}{ 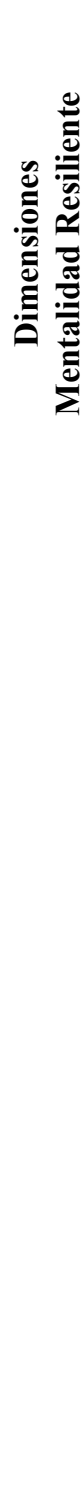 } & \multirow{3}{*}{$\begin{array}{l}\text { Diálogos de } \\
\text { vida. }\end{array}$} & & Bien CE & Dom. En. & Hab. Soc. & Bien. Fís. & $\begin{array}{l}\text { Autor } \\
\text { ef. }\end{array}$ \\
\hline & & Rho & $.324^{* *}$ & $.282^{* *}$ & $.379^{* *}$ & $.188^{* *}$ & $.404^{* *}$ \\
\hline & & $\begin{array}{l}\text { Sig. } \\
\text { (bilateral) }\end{array}$ & 0.000 & 0.000 & 0.000 & 0.004 & 0.000 \\
\hline & & Rho & $.346^{* *}$ & $.359^{* *}$ & $.426^{* *}$ & $.236^{* *}$ & $.294^{* *}$ \\
\hline & Empatía & $\begin{array}{l}\text { Sig. } \\
\text { (bilateral) }\end{array}$ & 0.000 & 0.000 & 0.000 & 0.000 & 0.000 \\
\hline & \multirow[b]{2}{*}{ Comunicación. } & Rho & $.326^{* *}$ & $.243^{* *}$ & $.354^{* *}$ & $.169^{* * *}$ & $.323^{* *}$ \\
\hline & & $\begin{array}{l}\text { Sig. } \\
\text { (bilateral) }\end{array}$ & 0.000 & 0.000 & 0.000 & 0.009 & 0.000 \\
\hline & \multirow{2}{*}{$\begin{array}{l}\text { Aceptación a sí } \\
\text { mismo y a los } \\
\text { demás. }\end{array}$} & Rho & $.481^{* *}$ & $.460^{* *}$ & $.431^{* *}$ & $.218^{* *}$ & $.436^{* *}$ \\
\hline & & $\begin{array}{l}\text { Sig. } \\
\text { (bilateral) }\end{array}$ & 0.000 & 0.000 & 0.000 & 0.001 & 0.000 \\
\hline & & Rho & $.272^{* *}$ & $.334^{* *}$ & $.495^{* *}$ & $.231^{* *}$ & $.343^{* *}$ \\
\hline & $\begin{array}{l}\text { Contactos y } \\
\text { compasión. }\end{array}$ & $\begin{array}{l}\text { Sig. } \\
\text { (bilateral) }\end{array}$ & 0.000 & 0.000 & 0.000 & 0.000 & 0.000 \\
\hline & \multirow{2}{*}{$\begin{array}{l}\text { Manejo de } \\
\text { errores. }\end{array}$} & Rho & $.289^{* *}$ & $.348^{* *}$ & $.340^{* *}$ & $.217^{* *}$ & $.291^{* *}$ \\
\hline & & $\begin{array}{l}\text { Sig. } \\
\text { (bilateral) }\end{array}$ & 0.000 & 0.000 & 0.000 & 0.001 & 0.000 \\
\hline & \multirow{2}{*}{$\begin{array}{l}\text { Manejo de } \\
\text { éxito. }\end{array}$} & Rho & $.421^{* *}$ & $.369^{* *}$ & $.407^{* *}$ & $.262^{* *}$ & $.362^{* *}$ \\
\hline & & $\begin{array}{l}\text { Sig. } \\
\text { (bilateral) }\end{array}$ & 0.000 & 0.000 & 0.000 & 0.000 & 0.000 \\
\hline & \multirow{2}{*}{$\begin{array}{l}\text { Autodisciplina } \\
\text { y autocontrol. }\end{array}$} & Rho & $.398^{* *}$ & $.390^{* *}$ & $.494^{* *}$ & $.290^{* *}$ & $.417^{* *}$ \\
\hline & & $\begin{array}{l}\text { Sig. } \\
\text { (bilateral) }\end{array}$ & 0.000 & 0.000 & 0.000 & 0.000 & 0.000 \\
\hline & \multirow{2}{*}{$\begin{array}{l}\text { Conocimiento } \\
\text { de una vida } \\
\text { resiliente }\end{array}$} & Rho & $.236^{* *}$ & $.218^{* *}$ & $.335^{* *}$ & $.307^{* *}$ & $.383^{* *}$ \\
\hline & & $\begin{array}{l}\text { Sig. } \\
\text { (bilateral) }\end{array}$ & 0.000 & 0.001 & 0.000 & 0.000 & 0.000 \\
\hline
\end{tabular}

Fuente: Elaboración propia (2017). 
En cuanto a la correlación entre las variables se presenta un coeficiente Rho de .615 considerada como positiva más cercana a moderada que a intensa (Tabla 2).

Tabla 2. Análisis de Correlación Rho de Spearman por variable

\begin{tabular}{|c|c|c|c|c|}
\hline \multirow{4}{*}{ 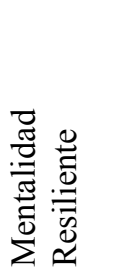 } & & & $\begin{array}{l}\text { Mentalidad } \\
\text { Resiliente }\end{array}$ & $\begin{array}{l}\text { Salud } \\
\text { Mental Positiva }\end{array}$ \\
\hline & $\begin{array}{l}\text { Coeficiente } \\
\text { correlación }\end{array}$ & de & 1.000 & $.615^{* *}$ \\
\hline & Sig. (bilateral) & & & .000 \\
\hline & $\mathrm{N}$ & & 238 & 238 \\
\hline \multirow{3}{*}{ 䲶 恶 } & $\begin{array}{l}\text { Coeficiente } \\
\text { correlación }\end{array}$ & de & $.615^{* *}$ & 1.000 \\
\hline & Sig. (bilateral) & & .000 & \\
\hline & $\mathrm{N}$ & & 238 & 238 \\
\hline
\end{tabular}

En el análisis de determinación presenta un coeficiente de .207, lo que permite explicar que la proporción de la variación total en la variable dependiente Mentalidad Resiliente, se explica por la variación en la variable independiente Salud Mental Positiva en un 20.70\%, es decir, la variable Salud Mental Positiva explica en este porcentaje la variación de la variable Mentalidad Resiliente, lo que refleja que en esta variable pueden estar incidiendo otros factores.

\section{Conclusiones}

Con base al objetivo general de la presente investigación que fue determinar la relación existente entre la Mentalidad Resiliente y la Salud Mental Positiva en los docentes de nivel superior, se concluye que los docentes de nivel superior de instituciones públicas (ciudad de Durango, Dgo. México) presentan una correlación moderada $\left(\mathrm{r}_{s}=.615\right)$, siendo la variable Salud Mental Positiva la que impacta o contribuye en el comportamiento de la variable dependiente Mentalidad Resiliente. El analizar y comprender la problemática existente entre los docentes ante las situaciones adversas que se le presentan puede permitir concientizar al propio docente sobre la importancia de desarrollar una Salud Mental Positiva que le permita llevar una vida resiliente en lo personal y en consecuencia de impacto positivo hacia su labor docente. 


\section{Referencias}

Barrera, M. L. (2011). Salud mental positiva: Conceptuación y construcción de un instrumento de medición para adultos. (Tesis de maestría no publicada), Universidad Autónoma de Yucatán, Mérida, México.

Barrera, M.L. \& Flores, M.M. (noviembre, 2013). Construcción de una Escala de Salud Mental Positiva para Adultos en Población Mexicana. Revista Iberoamericana de Diagnóstico y Evaluación - e Avaliacao Psicológica, 1(39). Recuperado de http://www.redalyc.org/pdf/4596/459645431003.pdf

Brooks, R. \& Goldstein, S. 2010). El poder de la resiliencia: Cómo lograr el equilibrio, la seguridad y la fuerza interior necesarios para vivir en paz. México: Paidós.

Cyrulnik, B. (2002). Los patitos feos. Barcelona, España: Gedisa.

Henderson, E. (1996). The international resilience project finding from the research and the effectiveness of interventions", Paper presented at the International Council of Psychologists, 54th, Banff, Canadá.

Henderson, N. \& Milstein, M. (2003). Resiliencia en la escuela. Argentina: Paidós.

Jahoda, M.T. (1958). Current Concepts of Positive Mental Health. Nueva York: Basic Books.

Lluch, M.T. (1999). Construcción de una escala para medir la salud mental positiva. (Tesis doctoral inédita). Facultad de Psicología. Barcelona: Universidad de Barcelona, España.

Organización Mundial de la Salud. (1990). La introducción de un componente de salud mental en la atención primaria. Ginebra: Organización Mundial de la Salud.

Organización Mundial de la Salud. (2004). Prevención de los trastornos mentales: Intervenciones efectivas y opciones de políticas públicas. Informe Compendiado.

Seligman, M.E. (2002). Authentic Happiness: Using the New Positive Psychology to Realize Your Potential for Lasting Fulfillment. New York: Free Press/Simon and Schuster.

Tengland, P. (2001). Mental Health: A philosophical analisys. Netherlands: Kluwer Academic Publishers.

Vanistendael, S. y Lecomte, J. (2002). La felicidad es posible. Despertar en niños maltratados la confianza en sí mismos: construir la resiliencia. España: Gedisa S.A

Vanistendael, S. (Julio, 2017). Apuntes inéditos. Diplomado Resiliencia aplicada. Reconstrucción desde la Adversidad. Universidad Juárez del Estado de Durango y Consultoría con Sentido Humano.

Wolin, S. \& Wolin, S. (1993). The resilient self: how survivors of troubled families rise above adversity. Nueva York, E.E.U.U.: Villard Books. 


\section{ANEXO 1}

\section{Cuestionario sobre Resiliencia y Salud Mental Positiva}

\section{Resiliencia}

4. Siempre, 3. Frecuentemente, 2. Pocas veces 1. Nunca

\begin{tabular}{|c|c|c|c|c|}
\hline \multirow{2}{*}{ Afirmaciones } & \multicolumn{4}{|c|}{ Respuestas } \\
\hline & 4 & 3 & 2 & 1 \\
\hline \multicolumn{5}{|l|}{$\begin{array}{l}\text { 1. Identifico guiones (pensamientos, creencias y comportamientos) negativos y me hago } \\
\text { responsable del cambio. }\end{array}$} \\
\hline \multicolumn{5}{|l|}{ 2. Puntualizo objetivos a corto y mediano plazo } \\
\hline \multicolumn{5}{|l|}{ 3. Considero posibles guiones nuevos o planes de acción que corresponden a los objetivos. } \\
\hline \multicolumn{5}{|l|}{ 4. Anticipo los posibles obstáculos que frenan el éxito y cómo podría superarlos. } \\
\hline \multicolumn{5}{|l|}{ 5. Cambio los objetivos, los guiones o el enfoque si el curso de acción seleccionado no resulta. } \\
\hline \multicolumn{5}{|l|}{ 6. Tengo empatía con mis alumnos en los momentos de disgusto, enfado, molestia o decepción. } \\
\hline \multicolumn{5}{|l|}{ 7. Aprecio y valoro el punto de vista de los demás. } \\
\hline \multicolumn{5}{|l|}{ 8. Aprecio cómo se percibe mi mensaje verbal. } \\
\hline \multicolumn{5}{|l|}{ 9. Aprecio cómo se percibe mi mensaje no verbal. } \\
\hline \multicolumn{5}{|l|}{ 10. Escucho activamente, entiendo y valoro lo que los demás están comunicando. } \\
\hline \multicolumn{5}{|l|}{ 11. Sé expresar mis sentimientos, pensamientos y creencias de forma verbal y no verbal. } \\
\hline \multicolumn{5}{|l|}{ 12. Tengo expectativas y objetivos realistas. } \\
\hline \multicolumn{5}{|l|}{ 13. Reconozco mis puntos fuertes. } \\
\hline \multicolumn{5}{|l|}{ 14. Reconozco mis puntos vulnerables. } \\
\hline \multicolumn{5}{|l|}{ 15. Hay correspondencia entre mis valores y objetivos. } \\
\hline \multicolumn{5}{|l|}{ 16. Tengo dos o tres personas que son adultos carismáticos en mi vida. } \\
\hline \multicolumn{5}{|l|}{ 17. Puedo ser un adulto carismático. } \\
\hline \multicolumn{5}{|l|}{ 18. Veo en los errores experiencias de aprendizaje y crecimiento personal. } \\
\hline \multicolumn{5}{|l|}{ 19. Evito comportamientos de defensa. } \\
\hline \multicolumn{5}{|l|}{$\begin{array}{l}\text { 20. Describo las islas de competencias o áreas de fortaleza para reforzarlas y ocuparlas en } \\
\text { futuros proyectos. }\end{array}$} \\
\hline \multicolumn{5}{|l|}{ 21. Tengo sentimiento de propiedad de éxito, experimento felicidad. } \\
\hline \multicolumn{5}{|l|}{ 22. Pienso antes de actuar. } \\
\hline \multicolumn{5}{|l|}{ 23. Tengo en cuenta los sentimientos de los demás. } \\
\hline \multicolumn{5}{|l|}{ 24. Realizo actividades divertidas. } \\
\hline \multicolumn{5}{|l|}{ 25. Añado humor a la vida. } \\
\hline \multicolumn{5}{|l|}{ 26. Conozco las características de los individuos resilientes. } \\
\hline \multicolumn{5}{|l|}{ 27. Conozco los obstáculos que pueden surgir para conseguir una Mentalidad Resiliente. } \\
\hline \multicolumn{5}{|l|}{$\begin{array}{l}\text { 28. Realizo ejercicios diarios y a largo plazo para mantener y reforzar una Mentalidad } \\
\text { Resiliente. }\end{array}$} \\
\hline 29. Tengo un plan de resiliencia para garantizar que el futuro esté lleno de satisfacciones y & & & & \\
\hline
\end{tabular}




\section{Salud mental positiva}

\section{Siempre, 3. Frecuentemente, 2. Pocas veces 1. Nunca}

\begin{tabular}{|c|c|c|c|c|}
\hline Afirmaciones & 4 & 3 & 2 & 1 \\
\hline 1. Me siento contento(a). & & & & \\
\hline 2. Me siento alegre. & & & & \\
\hline 3. Me siento feliz conmigo mismo (a). & & & & \\
\hline 4. Me siento bien con la vida que tengo. & & & & \\
\hline 5. Tengo una actitud positiva hacia la vida. & & & & \\
\hline 6. Me siento emocionalmente bien. & & & & \\
\hline 7. Puedo cuidarme solo(a) y defender mis derechos. & & & & \\
\hline 8. Necesito la aprobación de otras personas a la hora de tomar decisiones. & & & & \\
\hline 9. Actúo de acuerdo a mis propias convicciones. & & & & \\
\hline 10. Puedo solucionar con cierta tranquilidad los problemas de la vida diaria. & & & & \\
\hline 11. Me siento bien preparado(a) para enfrentar las situaciones difíciles de mi vida. & & & & \\
\hline 12. Soy una persona independiente. & & & & \\
\hline 13. Soy sociable. & & & & \\
\hline 14. Tengo facilidad para hacer amistades. & & & & \\
\hline 15. Me adapto fácilmente a diferentes situaciones sociales. & & & & \\
\hline 16. Puedo expresar lo que siento cuando estoy en un grupo con familiares, amigos o conocidos. & & & & \\
\hline 17. Puedo relacionarme positivamente con los y las demás. & & & & \\
\hline 18. Puedo expresar mi afecto sin dificultad. & & & & \\
\hline 19. Hago ejercicio físico (deportes, baile, correr, caminar, gimnasio, etc... & & & & \\
\hline 20. Me siento bien con la salud que tengo. & & & & \\
\hline 21. Tengo tiempo libre para hacer otras actividades diferentes a mis ocupaciones diarias. & & & & \\
\hline 22. Siento que mi organismo funciona de manera aceptable para mí. & & & & \\
\hline $\begin{array}{l}\text { 23. Realizo alguna actividad sana que me ayuda a desahogarme, relajarme o expresar mis } \\
\text { emociones. }\end{array}$ & & & & \\
\hline 24. Trato de cuidarme y llevar una alimentación sana. & & & & \\
\hline 25. Me analizo a mí mismo(a). & & & & \\
\hline 26. Hablo conmigo, me pregunto qué quiero, qué siento. & & & & \\
\hline 27. Reflexiono acerca de mí. & & & & \\
\hline $\begin{array}{l}\text { 28. Después de hacer algo importante para mí, pienso acerca de lo que hice, cómo lo hice o por } \\
\text { qué lo hice. }\end{array}$ & & & & \\
\hline 29. Conozco mis virtudes, habilidades y defectos. & & & & \\
\hline 30. Analizo los problemas cuando los enfrento. & & & & \\
\hline
\end{tabular}

